\title{
Machine Learning Enabled Traveltime Inversion Based on the Horizontal Source Location Perturbation
}

\author{
Isa Eren Yildirim*, Tariq Alkhalifah ${ }^{\dagger}$, Ertugrul Umut Yildirim* \\ *Institute of Applied Mathematics \\ Middle East Technical University \\ Üniversiteler Mahallesi, Dumlupınar Bulvarı \\ Çankaya/Ankara, 06800, Turkey \\ yildirim.isa@metu.edu.tr; ertugrul.yildirim@metu.edu.tr \\ ${ }^{\dagger}$ Physical Sciences and Engineering Division \\ King Abdullah University of Science and Technology \\ 4700 KAUST \\ Thuwal, 23955, KSA \\ tariq.alkhalifah@kaust.edu.sa
}

(October 7, 2021)

GEO-2020-0735.R3

Running head: ML Enabled Traveltime Inversion

\begin{abstract}
Gradient based traveltime tomography, which aims to minimize the difference between modeled and observed first arrival times, is a highly non-linear optimization problem. Stabilization of this inverse problem often requires employing regularization. While regularization helps avoid local minima solutions, it might cause low resolution tomograms because of its inherent smoothing property. On the other hand, although conventional ray-based tomog-
\end{abstract}


raphy can be robust in terms of the uniqueness of the solution, it suffers from the limitations inherent in ray tracing, which limits its use in complex media. To mitigate the aforementioned drawbacks of gradient and ray-based tomography, we approach the problem in a novel way leveraging data-driven inversion techniques based on training deep convolutional neural networks (DCNN). Since DCNN often face challenges in detecting high level features from the relatively smooth traveltime data, we use this type of network to map horizontal changes in observed first arrival traveltimes caused by a source shift to lateral velocity variations. The relationship between them is explained by a linearized eikonal equation. Construction of the velocity models from this predicted lateral variation requires information from, for example, a vertical well-log in the area. This vertical profile is then used to build a tomogram from the output of the network. Both synthetic and field data results verify that the suggested approach estimates the velocity models reliably. Because of the limited depth penetration of first arrival traveltimes, the method is particularly favorable for near-surface applications. 


\section{INTRODUCTION}

First arrival traveltime picking and inversion for near-surface velocity structures has been widely used in seismic exploration (Zhang and Toksöz, 1998; Zhu et al., 1992; Taillandier et al., 2009). The quality of seismic images highly depends on the velocity model building and remains a challenge especially when the complexity of the near-surface is unilluminated. Hence, effectively detecting velocity changes in the near-surface is crucial for delineating deeper structures in depth imaging. The governing mathematical model that describes the relationship between the first-break traveltimes and the wavefront propagation velocities is a non-linear eikonal partial differential equation (PDE). The linearized version of the eikonal equation, on the other hand, linearly relates small changes in traveltime to small changes in velocity and its connection with traveltime tomography were previously addressed by Aldridge (1994), Fomel (2001) and Alkhalifah and Fomel (2010).

With the advancement in computing power in recent years, machine learning (ML) has found a place in seismic data processing and inversion tasks utilizing its ability to map from data space to model space by detecting implicit relations between previously supplied inputs and outputs. For example, Araya-Polo et al. (2018) used deep learning (DL) to predict velocity models from recorded seismic data. Wang et al. (2018) applied deep convolutional neural networks for velocity model building directly from raw seismograms. In the semblance analysis based velocity model building, Park and Sacchi (2020) recently used DL to predict stacking velocities from the semblance panels. There were also few attempts to improve traveltime tomograms by incorporating machine learning to traveltime inversion problems. Li et al. (2019) developed a constrained optimization problem based on the Generative Adversarial Network (GAN). Guo et al. (2019) combined classical gradient based traveltime 
tomography with a supervised descent method (SDM). Earp and Curtis (2020) proposed neural network based Bayesian traveltime inversion for $2 \mathrm{D}$ velocity predictions.

In this study, we revisit the linearized eikonal equation for velocity estimation within the context of machine learning. Our aim is to teach a convolutional neural network $(\mathrm{CNN})$ to learn the connection between horizontal derivatives of the traveltimes and horizontal derivatives of velocities. This approach is entirely data driven and enables us to estimate the lateral variations of the model without needing conventional ray-based methods as well as gradient based algorithms. With the help of well information from the area, the tomogram can be constructed from the output of the network.

This paper is organized as follows. First, the theory of the linearized eikonal equation and its usage in an inversion framework is provided. Then, we describe how we create the training data and explain the network architecture. To evaluate our new approach, then we test it on synthetic as well as field data. Finally, in the conclusions section, we summarize our study.

\section{THEORY}

In a $2 \mathrm{D}$ medium represented by dimensions $\{x, z\}$ with slowness squared (sloth), $w$, the traveltime, $T$ computation amounts to solving the following non-linear PDE:

$$
\left(\frac{\partial T}{\partial x}\right)^{2}+\left(\frac{\partial T}{\partial z}\right)^{2}=w
$$

By assuming the sloth field is continuous in the horizontal direction, equation 1 can be converted to a linear form by taking its derivative with respect to a lateral source shift $s$ 
(Alkhalifah and Fomel, 2010):

$$
\nabla T \cdot \nabla \frac{\partial T}{\partial s}=-\frac{1}{2} \frac{\partial w}{\partial x}
$$

Note that the negative sign at the right hand side of equation 2 arises from the fact that a source shift in one direction is equivalent to shifting the velocity model in the opposite direction. Equation 2 means that with a known traveltime derivative along the horizontal direction, lateral velocity variations can be predicted.

Denoting the solution of equation 2 as $D T$ and the derivative along the $x$-axis of sloth as $D w$, the objective function for an optimization problem from the deterministic point of view can be written as

$$
F(D w)=\frac{1}{2}\left\|D T-D T^{o b s}\right\|_{2}^{2}
$$

where $D T^{o b s}$ represents the observed traveltime derivatives along the source shifting direction. $\|\cdot\|_{2}^{2}$ is the $L_{2}$ norm taken in Euclidean space.

Whereas, from the statistical learning aspect, which is the focus of this work, the aim becomes minimizing the pixel-wise error between the velocity derivatives supplied to the network (the labels) and the CNN predicted ones. Considering the loss function as the mean squared error (MSE), which is commonly used for regression type problems, the objective of the training process can be given for an $N$ number of instances as

$$
L(\varphi)=\frac{1}{N} \sum_{n=1}^{N}\left(D V_{i}-D V_{i}^{r}\right)^{2}
$$

where $D V_{i}$ is the $i$ th target model in terms of the lateral derivative of velocity, $D V_{i}^{r}$ stands for the $i$ th output model of the ML tomography operator $f\left(D T_{i}, \varphi\right)$, which takes the $i$ th image of the traveltime derivatives as input. Here, $\varphi$ are the weights of the network, which 
are iteratively updated with the corresponding gradients of the loss function as follows:

$$
\varphi_{k+1}=\varphi_{k}-\alpha \frac{\partial L}{\partial \varphi_{k}}
$$

Here, $\alpha$ denotes the learning rate.

The operator obtained from the minimized loss function is then used for estimating velocity derivatives $D V(x, z)$ as an important step to build a tomogram $V(x, z)$ using a known vertical profile $V_{0}(z)$ as follows:

$$
V(x, z)=V_{0}(z)+\int D V(x, z) d x
$$

\title{
THE NUMERICAL IMPLEMENTATION
}

\begin{abstract}
We start by establishing the network and performing the training of such a network. We follow that by testing the network on traveltime computed synthetically and others from field data.
\end{abstract}

\section{Preparation of training data and network properties}

Our deep learning approach to traveltime tomography is based on supervised learning and requires creating a set of training data (features and labels). As our focus in this work is the near-surface, we randomly generated velocity models for that purpose. The random models are created as follows. Firstly, a portion containing a normal fault of the Hess VTI velocity model is chosen as a base model. We created a model with fault systems (reverse, normal, or both) by flipping and replicating random crops from the base. Next, we applied an elastic distortion technique, which originally was used for expanding the training data for handwritten digit recognition tasks (Simard et al., 2003). In this technique, displacement 
fields are created from a Gaussian random field and then we apply them to images to deform them. The standard deviation of the Gaussian controls the smoothness of deformation, while the scaling factor that is multiplied by the fields arranges the intensity of the deformation. We applied vertical distortion which inserts folding and possible layer repositioning to a model. Finally, by cropping random portions from the distorted model and resizing them to the desired size, we managed to generate a set of models that may represent the nearsurface. A similar pseudo-random model generation methodology was previously used for a DL application that aims to estimate velocity profiles from the seismic data (Kazei et al., 2021). The generated models might reflect a certain geological composition of the base model and may not be appropriate to use for all purposes. However, with the help of a known vertical profile from a region (like from a well), it would be possible to create training data containing different geological structures (caves, fissures, etc.) that can serve one's needs using the technique we described.

The mean and standard deviation of the 4400 generated velocity models used for training are presented in Figure 1. The mean of the models shows general smooth layered media with velocity gradually increasing from 2.6 to nearly $3.5 \mathrm{~km} / \mathrm{s}$. The standard deviation, on the other hand, indicates that the velocities deviate less from the mean with increasing depth. Such features of the training set serve our purpose, as velocities tend to increase with depth, and the resolution of our inversion methods tend to decrease with depth.

Traveltime responses from all models are obtained by equation 1. Surface traveltimes from each shot are placed in rows to form a traveltime image with horizontal dimension representing the receiver and the vertical direction representing the shot dimension. Figure 2 shows the guiding model, a sample from randomly generated models and its corresponding traveltime image. Since our intention is to map traveltime lateral derivatives to lateral 
velocity changes, we need to compute the traveltime derivatives to train our model. Once the surface traveltimes for each shot are obtained, it is easy to compute the horizontal traveltime derivatives. For that, each successive shot locations are superimposed, the corresponding traveltimes are subtracted and then divided by the shot distance. Four representative input images and their corresponding outputs from the training data are displayed in Figure 3.

[Figure 1 about here.]

[Figure 2 about here.]

Using the data we train a CNN. The CNN architecture used in this work is a fully convolutional network (FCN). It is a good choice for inversion purposes that require high dimensional $2 \mathrm{D}$ or $3 \mathrm{D}$ outputs. Since choosing the optimal hyperparameters that define the network model is often problem specific, we arrived to our model settings by examining the learning curves for different settings. The encoder stage of the FCN architecture consists of three sets of convolutional blocks with max pooling layers. Similarly, the decoder stage contains three sets of convolutional blocks with upscaling layers (Figure 4). In each block; convolution, batch normalization, and leaky ReLU activation functions are used, respectively. The number of filters starts from 32 and increases up to 512 in the encoder stage, while in the decoder stage, we decrease the number of filters back to 32 before the networks's prediction of the velocity derivatives. Additionally, dropout layers are used after each convolutional block to prevent overfitting. Our implementation of CNN using the TensorFlow and Keras open-source libraries is carried out using Google's tensor processing units (TPU).

[Figure 3 about here.] 
[Figure 4 about here.]

\section{Synthetic example}

For the synthetic training and testing, 4800 data examples with a size of $64 \mathrm{x} 256$ were used corresponding to 4800 augmentations of the velocity model. To find the corresponding traveltimes, the model was sampled $\Delta=8 \mathrm{~m}$ in both the horizontal and vertical directions. 64 shots were evenly distributed on the surface and receivers were placed on each sample point, which makes the maximum receiver offset equal to $2.04 \mathrm{~km}$. A total of 400 of the generated examples were separated as test data that were not seen by the network during the training. The remaining 4400 models were fed into the neural network for training, and thus, 400 models were reserved for the validation data. The inputs of the network, i.e., traveltime derivatives, vary from -0.5 to 0.3 . Since the range of the data is relatively small and rescaling can reduce the feature extraction ability of the network in this particular case, normalization of the data was not performed before the training.

The mean absolute error (MAE) corresponding to the $L_{1}$ norm was chosen as the loss function. Mean squared error was also used as a metric to track the progress of the errors. Based on several trial experiments, the CNN was trained with the Adam optimizer with a batch size of 16 . The learning rate starts from 0.01 and decreases by a factor of 0.1 when the model performance reaches a plateau. An epoch takes nearly 10 seconds though the network has 10.9 million trainable parameters. Although the epoch number was set to be 250 for the training, no further improvement was seen after around 140 epochs paving the way to stop the training early. We observed that both the training and the validation errors decrease during the training. While the training errors for both metrics decreased 
monotonically, the validation error for MAE dropped from 0.36 to 0.20 and around 1.00 to 0.40 for MSE with some fluctuations (Figure 5).

Once the network was trained, the model was ready for testing. Previously reserved test data were used to measure the performance of our trained network. In Figure 6, we can see a few labels from the test data and corresponding estimations made by the neural network. The lateral velocity derivative errors for each sample are also provided. Sharp transitions in the lateral velocity changes have been effectively detected by our network model, which allows us to accurately predict discontinuities such as faults and folds.

[Figure 5 about here.]

[Figure 6 about here.]

As the output of the network is a derivative of velocity with respect to the lateral direction, we need to construct velocity models by integrating the output along the horizontal direction. Assuming we know the velocity along a vertical profile from the middle of the domain, velocity images can easily be generated from the derivative information. In Figure 7, we show the results from four predictions along with their true counterparts. Moreover, conventional traveltime tomography was implemented for the selected four velocity models (Figure 8). Starting models for the inversion were 2D extensions of the vertical profiles that are utilized to build tomograms from the CNN outputs. The conjugate-direction (CD) method (Claerbout, 2014) was performed for all the experiments. A total of 5 linearization steps each having 20 conjugate gradient iterations were applied. After the fifth linearization update, no further improvements were observed in the $l_{2}$ data misfit. As expected, all the inverted models resulted in very smooth representations of the true ones. On the other 
hand, in the ML-based results, not only the shallow parts of the models were captured but also the deeper parts were satisfyingly recovered. The performances can also be compared by investigating the percent error maps shown in the last column of Figure 7 and Figure 8 . Though the errors between the ground truth and the estimated models in our method are negligible compared to the errors in the classical tomograms, one may wonder about the lateral artifacts in the recovered models. These horizontal imprints in the inverted models are caused by the nature of the approach we adopted, that is to say when the true derivatives could not be perfectly captured by the neural network the errors propagate in the direction of integration to build the velocity. This explains some noticeable distortions in the velocity models in the second and fourth rows of Figure 7. To compare the results for conventional and ML-based inverted models, we provide the vertical velocity profiles in Figure 9. The results demonstrate the accuracy of our network for the test data by producing accurate velocity predictions.

[Figure 7 about here.]

[Figure 8 about here.]

[Figure 9 about here.]

\section{A field data example}

We then applied the method to a field data set that was acquired from Beiya Town, China. The study area contains ore deposits coming from the Himalayan alkali-rich porphyry (Zou and Mao, 2014). We use a 2D dataset from the region (Figure 10). The acquisition geometry consists of 17 shots with an irregular interval and 401 receivers for each shot fully covering 
the $8 \mathrm{~km}$ line, which results in a receiver interval of $20 \mathrm{~m}$. For the seismic trace, the number of time samples is 4001 with a time sample interval of $2 \mathrm{~ms}$.

[Figure 10 about here.]

Using source receiver reciprocity and interpolation, we rearrange the geometry so that we have more shots, which are evenly spaced on the surface. We use 201 shots with a source spacing of $40 \mathrm{~m}$ and 801 receivers for each shot on the survey area. We obtained the interpolated traveltimes (Figure 11) using a simple Artificial Neural Network (ANN) containing just two hidden layers. The input data for the ANN is lateral coordinates of the sources and receivers. The fully connected hidden layers are composed of 128 and 256 neurons, respectively, and the rectified linear unit (ReLU) is used as an activation for each node. The output is a single node representing the traveltime. The ANN performance is evaluated by MAE.

[Figure 11 about here.]

The acquisition, chosen for the field data, is also applied to the velocity models we used in the CNN training for the sake of consistency. The architecture and hyperparameters of the model are the same as that of the synthetic data training. As the range of the field data derivatives are different from the training set, the input values are rescaled between 0 and 1 before the training and the MSE is used as a loss function. The trained CNN model is then used to estimate the lateral velocity variations in the interested region. Figure 12 shows the input and output of the network. Traditional tomographic reconstruction performed with the CD method using the field data is also carried out and presented in Figure 13 for comparison with the ML-based inverted model. 
[Figure 12 about here.]

An acceptable vertical velocity profile (Kai Chang, personal communication, 2020) from $4.6 \mathrm{~km}$ is utilized to build an inverted velocity model (Figure 13(c)). The 2D extension of the same profile is used as an initial model for conventional tomography. The tomographic inversion result based on our approach as opposed to the traditional one (Figure 13(b)) is consistent with the geological section (Figure 13(a)), which presumably formed by assumptions from other studies conducted around the study area. Two high-velocity structures, possibly ore-bearing porphyry deposits, at the right and the left part of the geological model are captured by the tomogram. Also, the high-velocity intrusion zone, which may include alkali-rich content, which stretches from the left bottom to the right upper part can be tracked on the inverted model as well as on the velocity derivative image.

Though solving the eikonal equation does not require a lot of computational resources, let's say, when compared to full-wave modeling, arranging the acquisition consistent with the field data and solving forward problems for each velocity model, then retraining a network might be seen as a challenge for practitioners. For a much larger training dataset than the one we used in this study, this issue can be tackled by using transfer learning, a technique utilizing the stored knowledge from a pre-trained network model. In doing so, both the computational costs of forward modeling and training stages can be significantly reduced in field data applications.

[Figure 13 about here.] 


\section{CONCLUSIONS}

We developed a CNN based approach for traveltime inversion by using the relation between the observed traveltime changes and subsurface velocity variations. The synthetic test as well as the field data result verified that the FCN architecture is successful in capturing abstractions from data that contains traveltime variations and can be used to accurately estimate the velocity model, as compared to traditional tools, especially when the geological focus is the near-surface. Since we are dealing with traveltimes, the field example demonstrated its portability and flexibility. We note that there is a need for a proper training dataset reflecting the geology of the investigated area. This can be achieved by the data generation technique we described earlier using a vertical profile from a region of interest. Future research could look into the application of the proposed methodology to 3D tomograms or could consider anisotropy for more reliable macro-velocity model estimations.

\section{ACKNOWLEDGMENTS}

We thank Vladimir Kazei for his useful suggestions. We also would like to thank Prof. Qiusheng Li for providing the seismic data as well as Kai Chang for sharing the vertical velocity profile and first arrival times.

14 


\section{REFERENCES}

Aldridge, D. F., 1994, Linearization of the eikonal equation: Geophysics, 59, 1631-1632.

Alkhalifah, T., and S. Fomel, 2010, An eikonal-based formulation for traveltime perturbation with respect to the source location: Geophysics, 75, T175-T183.

Araya-Polo, M., J. Jennings, A. Adler, and T. Dahlke, 2018, Deep-learning tomography: The Leading Edge, 37, 58-66.

Claerbout, J., 2014, Geophysical image estimation by example: www.lulu.com.

Earp, S., and A. Curtis, 2020, Probabilistic neural network-based 2d travel-time tomography: Neural Computing and Applications, 32, 1-19.

Fomel, S., 2001, Traveltime computation with the linearized eikonal equation: SEP, 94.

Guo, R., M. Li, S. Xu, and A. Abubakar, 2019, First arrival traveltime tomography using supervised descent learning technique: Inverse Problems, 35.

Kazei, V., O. Ovcharenko, P. Plotnitskii, D. Peter, X. Zhang, and T. Alkhalifah, 2021, Mapping full seismic waveforms to vertical velocity profiles by deep learning: Geophysics, 86, R711-R721.

Li, Z., X. Jia, and J. Zhang, 2019, Deep learning guiding first-arrival traveltime tomography: 89th Annual International Meeting, SEG, Expanded Abstract, 2513-2517.

Park, M. J., and M. D. Sacchi, 2020, Automatic velocity analysis using convolutional neural network and transfer learning: Geophysics, 85, 1JF-Z3.

Simard, P., D. Steinkraus, and J. Platt, 2003, Best practices for convolutional neural networks applied to visual document analysis: Seventh International Conference on Document Analysis and Recognition, 2003. Proceedings., IEEE Comput. Soc, 958-963.

Taillandier, C., M. Noble, H. Chauris, and H. Calandra, 2009, First-arrival traveltime tomography based on the adjoint-state method: Geophysics, 74, WCB1-WCB10. 
Wang, W., F. Yang, and J. Ma, 2018, Velocity model building with a modified fully convolutional network: 88th Annual International Meeting, SEG, Expanded Abstract, 20862090.

Zhang, J., and M. Toksöz, 1998, Nonlinear refraction traveltime tomography: Geophysics, 63, $1726-1737$.

Zhu, X., D. P. Sixta, and B. G. Angstman, 1992, Tomostatics: Turning-ray tomography + static corrections: The Leading Edge, 11, 15-23.

Zou, G., and Y. Mao, 2014, The geological characteristics and genesis of beiya goldpolymetallic deposit, yunnan province, china: Acta Geologica Sinica - English Edition, 88, $672-675$. 


\section{LIST OF FIGURES}

1 Basic statistical characteristics of the dataset that is used for training. The mean of the 4400 training models (a), and the standard deviation of the 4400 training models $(\mathrm{b}) . \ldots \ldots \ldots \ldots$

2 A portion of Hess velocity model (a), an example from the simulated models (b), its corresponding traveltime image $(\mathrm{c}) \ldots \ldots \ldots$

3 Four randomly selected examples from the set of input data (a), and the corresponding ground truth velocity derivative models $(\mathrm{b}) \ldots \ldots . . .$. .

4 The FCN architecture mapping lateral traveltime derivatives to lateral velocity derivatives. Each box represents a feature map. The number above each box indicates the number of channels and the dimensions are shown in the bottom of each box. . . . . . . . . . . . . . . . . . .

5 Mean absolute error (MAE) loss function curve for both training and validation sets (a), mean squared error (MSE) evaluation metric curve for both training and validation sets $(\mathrm{b}) \ldots \ldots \ldots \ldots$

6 The first column shows four examples of true velocity derivatives (groundtruth) in the horizontal direction from the test data; the second column shows the corresponding outputs of the network; the last column shows the difference between the true and the prediction velocity derivatives. . . . . .

$7 \quad$ The first column shows true velocity models for the four selected examples in Figure 6 ; the second column shows the estimated velocity models built from assumed well measurements taken from the middle of the true models; the last column shows the percentage error maps between the true model and the prediction. . . . . . . . . . . . . . . .

8 The first column shows true velocity models for the four selected examples in Figure 7; the second column shows estimated velocity models from conventional tomography; the last column shows the percentage error maps between the true model and the tomography result. . . . . . . . . . . .

$9 \quad$ Vertical velocity profiles of the selected models in Figures 7 and 8 at $1.2 \mathrm{~km}$.

10 Location map of the study area. The yellow line is where the acquisition was conducted, and the red stars denote the shot positions (Courtesy of Kai Chang). . . . . . . . . . . . . . . . . .

11 The interpolated traveltimes for the field data. The traveltimes were interpolated into 201 shots and 801 receivers for each shot. . . . . . . . . .

12 The field data derivative image (a). The first arrival time derivatives computed using two successive shots form the rows of the image. CNN based predicted lateral velocity variations $(b) . \ldots \ldots \ldots \ldots$

13 A geological section representing the region of interest (Courtesy of Kai Chang, University of Science and Technology of China) (a). The inverted model from conventional tomography (b). A CNN predicted model (c). . . 


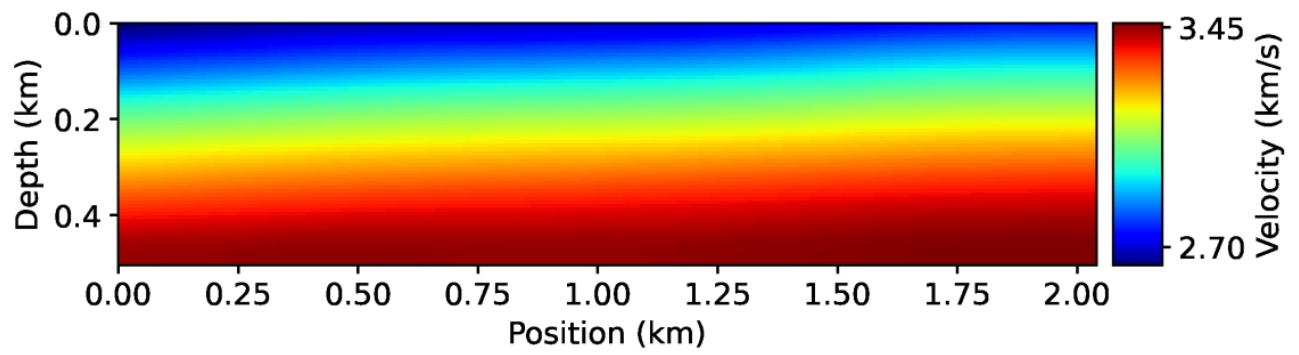

1a. Basic statistical characteristics of the dataset that is used for training. The mean of the 4400 training models (a), and the standard deviation of the 4400 training models(b).

$499 \times 142 \mathrm{~mm}(118 \times 118 \mathrm{DPI})$ 


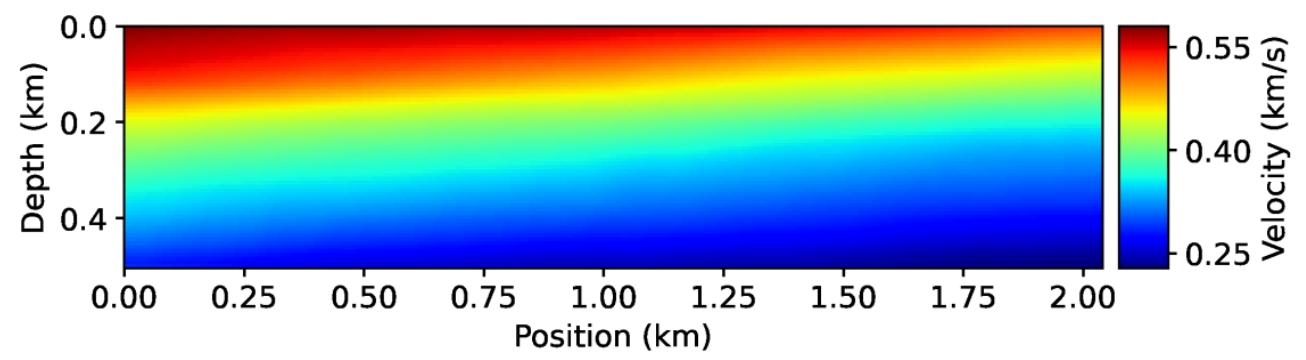

1b. Basic statistical characteristics of the dataset that is used for training. The mean of the 4400 training models (a), and the standard deviation of the 4400 training models(b).

$499 \times 142 \mathrm{~mm}(118 \times 118 \mathrm{DPI})$ 


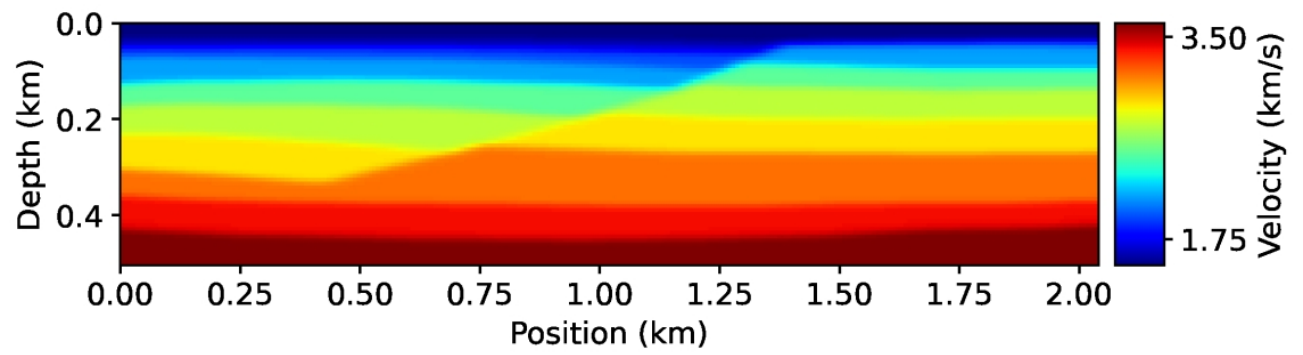

2a. A portion of Hess velocity model (a), an example from the simulated models (b), its corresponding traveltime image(c).

$499 \times 142 \mathrm{~mm}(118 \times 118 \mathrm{DPI})$ 


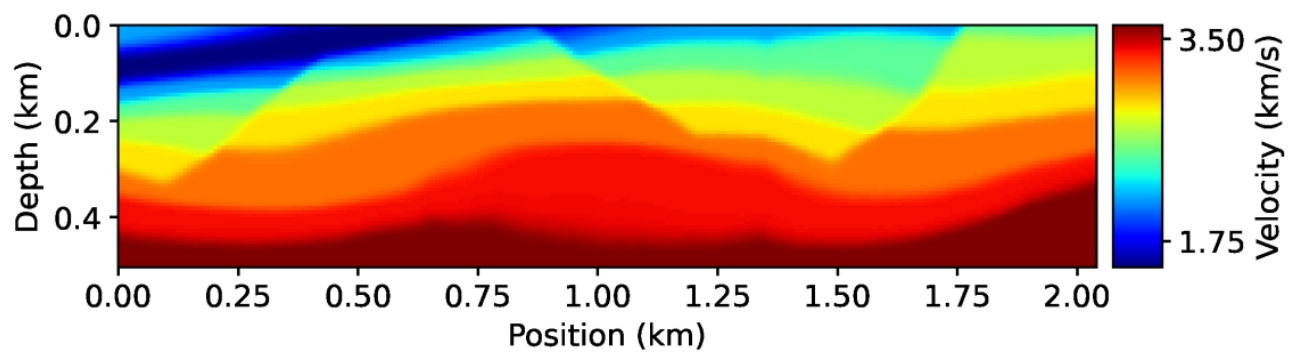

2b. A portion of Hess velocity model (a), an example from the simulated models (b), its corresponding traveltime image(c).

$499 \times 142 \mathrm{~mm}(118 \times 118 \mathrm{DPI})$ 


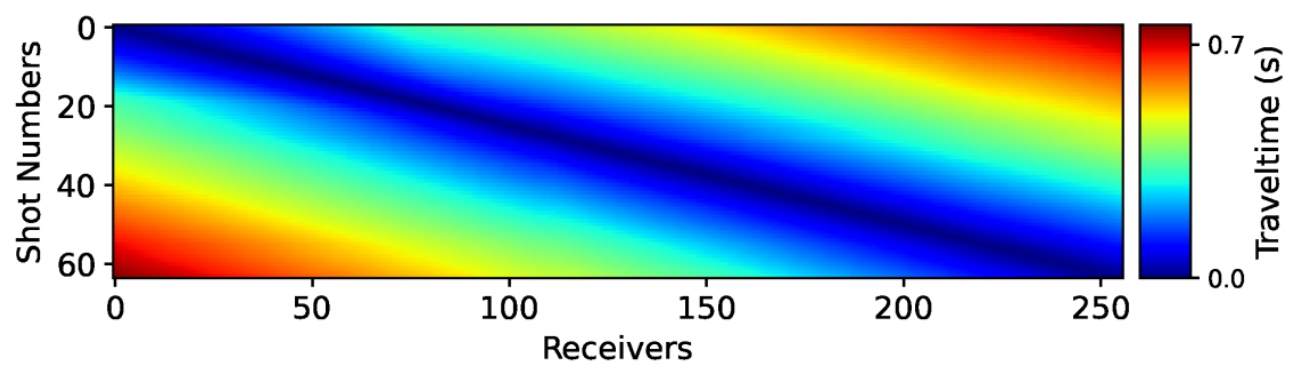

2c. A portion of Hess velocity model (a), an example from the simulated models (b), its corresponding traveltime image(c).

$484 \times 142 \mathrm{~mm}(118 \times 118 \mathrm{DPI})$ 

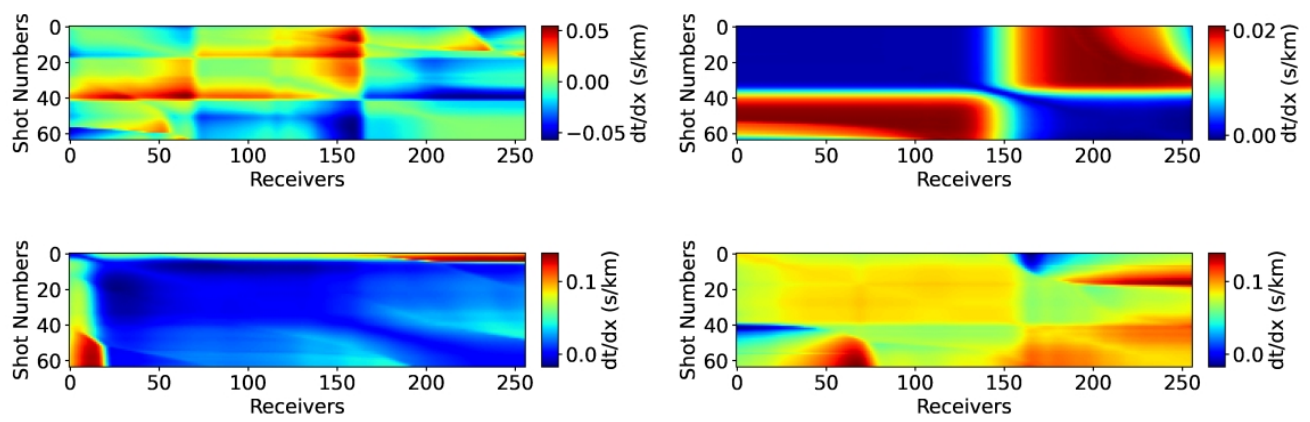

3a. Four randomly selected examples from the set of input data (a), and the corresponding ground truth velocity derivative models (b).

$1066 \times 344 \mathrm{~mm}(118 \times 118$ DPI $)$ 

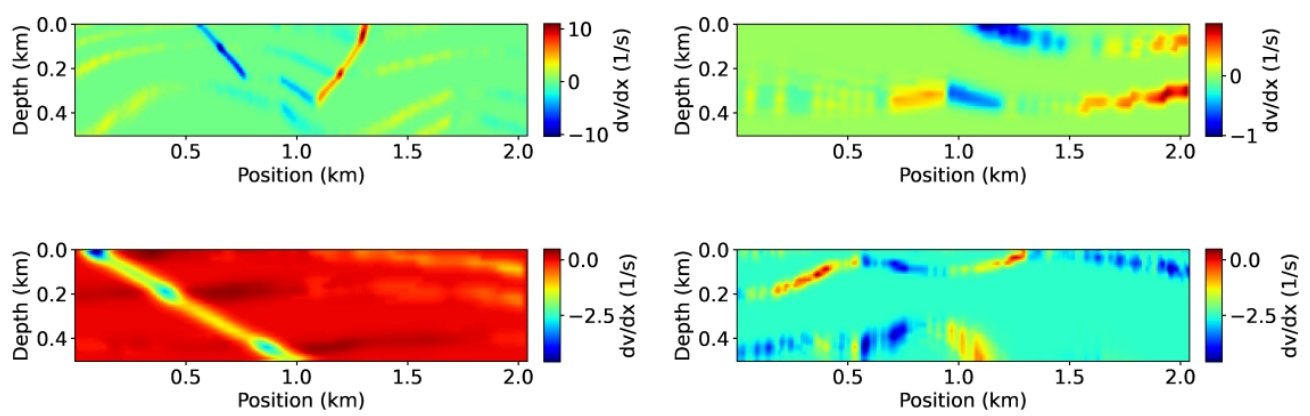

3b. Four randomly selected examples from the set of input data (a), and the corresponding ground truth velocity derivative models $(b)$.

$1074 \times 338 \mathrm{~mm}(118 \times 118 \mathrm{DPI})$ 


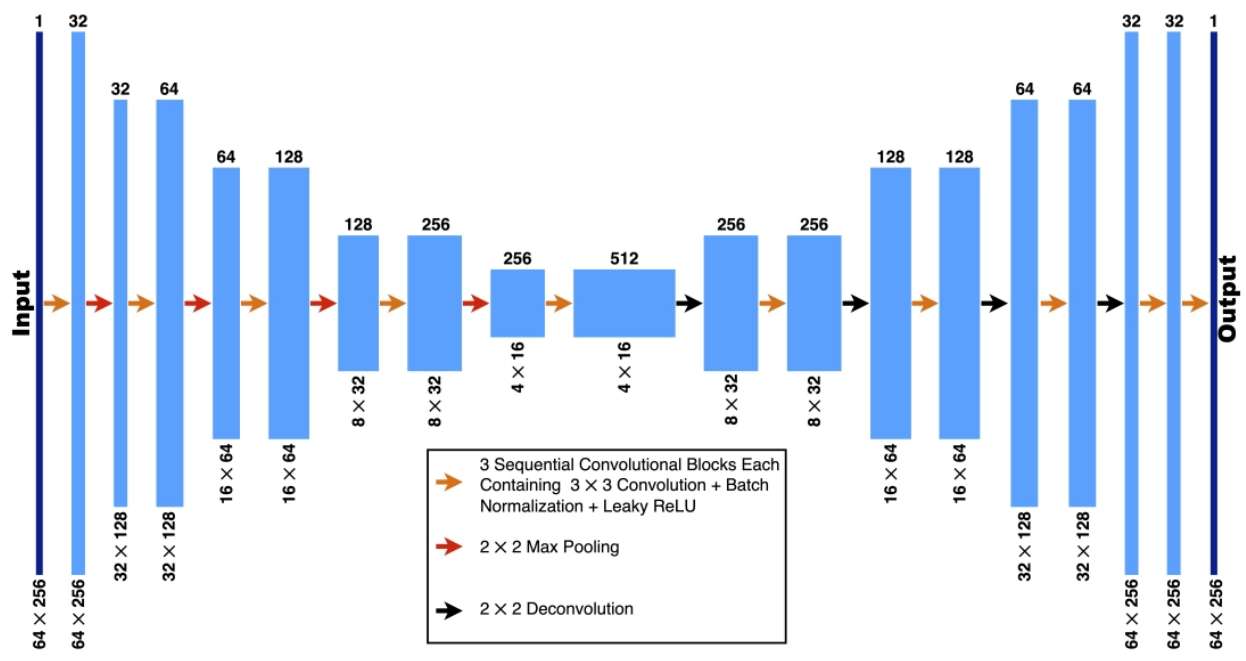

4 The FCN architecture mapping lateral traveltime derivatives to lateral velocity derivatives. Each box represents a feature map. The number above each box indicates the number of channels and the dimensions are shown in the bottom of each box.

$677 \times 381 \mathrm{~mm}(300 \times 300 \mathrm{DPI})$ 


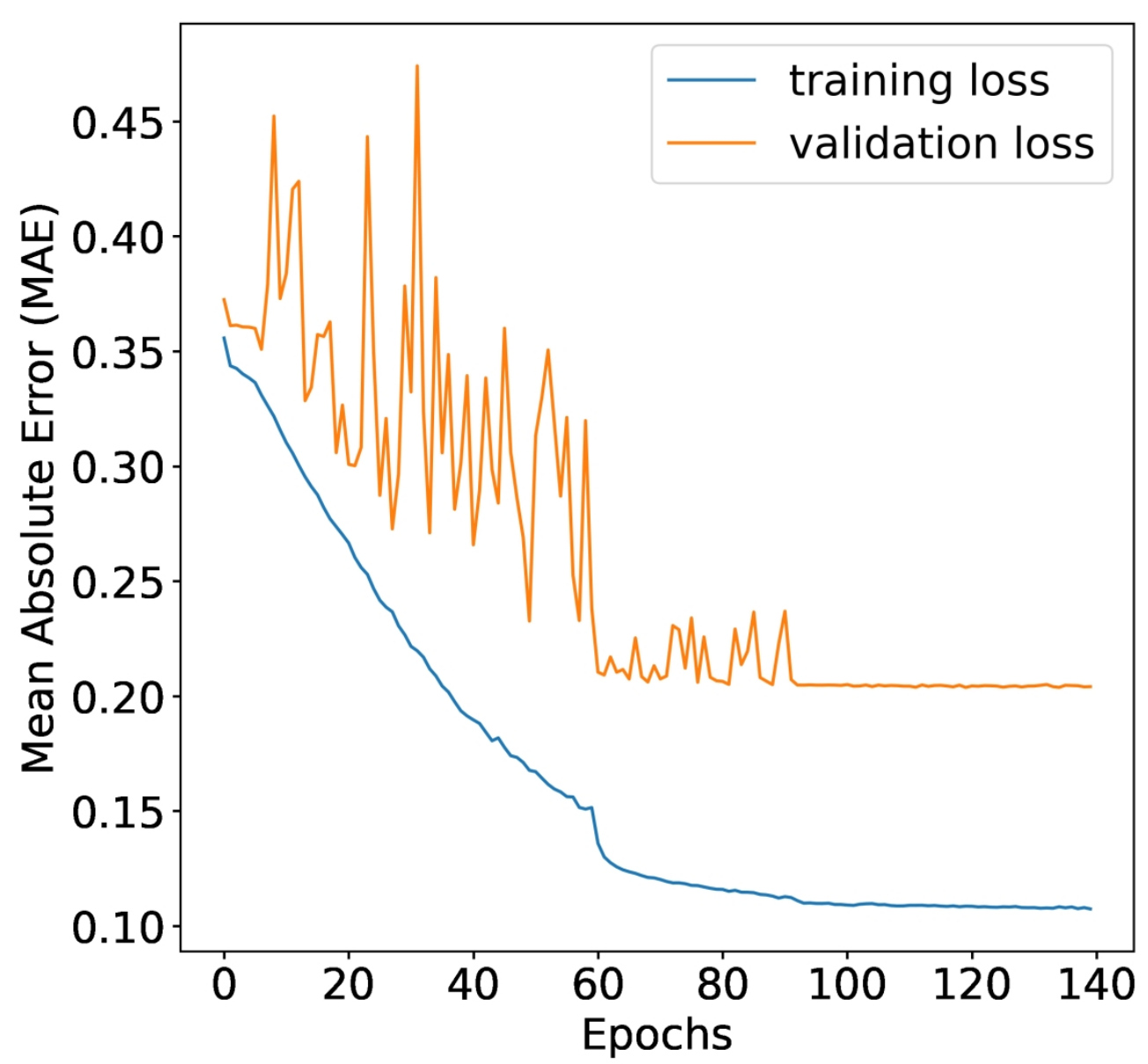

5a. Mean absolute error (MAE) loss function curve for both training and validation sets (a), mean squared error (MSE) evaluation metric curve for both training and validation sets(b).

$481 \times 447 \mathrm{~mm}(118 \times 118 \mathrm{DPI})$ 


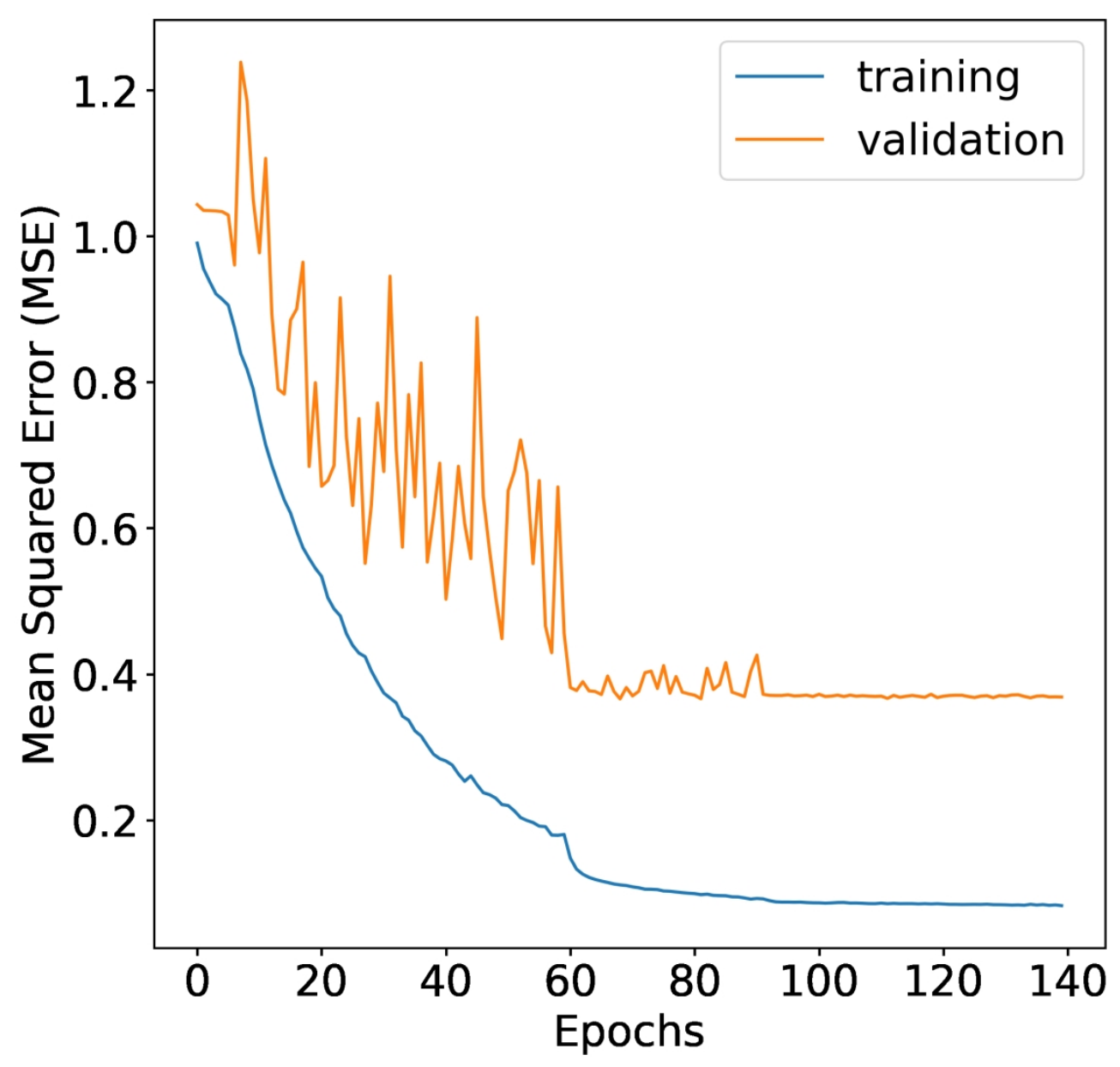

5b. Mean absolute error (MAE) loss function curve for both training and validation sets (a), mean squared error (MSE) evaluation metric curve for both training and validation sets(b).

$470 \times 447 \mathrm{~mm}(118 \times 118 \mathrm{DPI})$ 

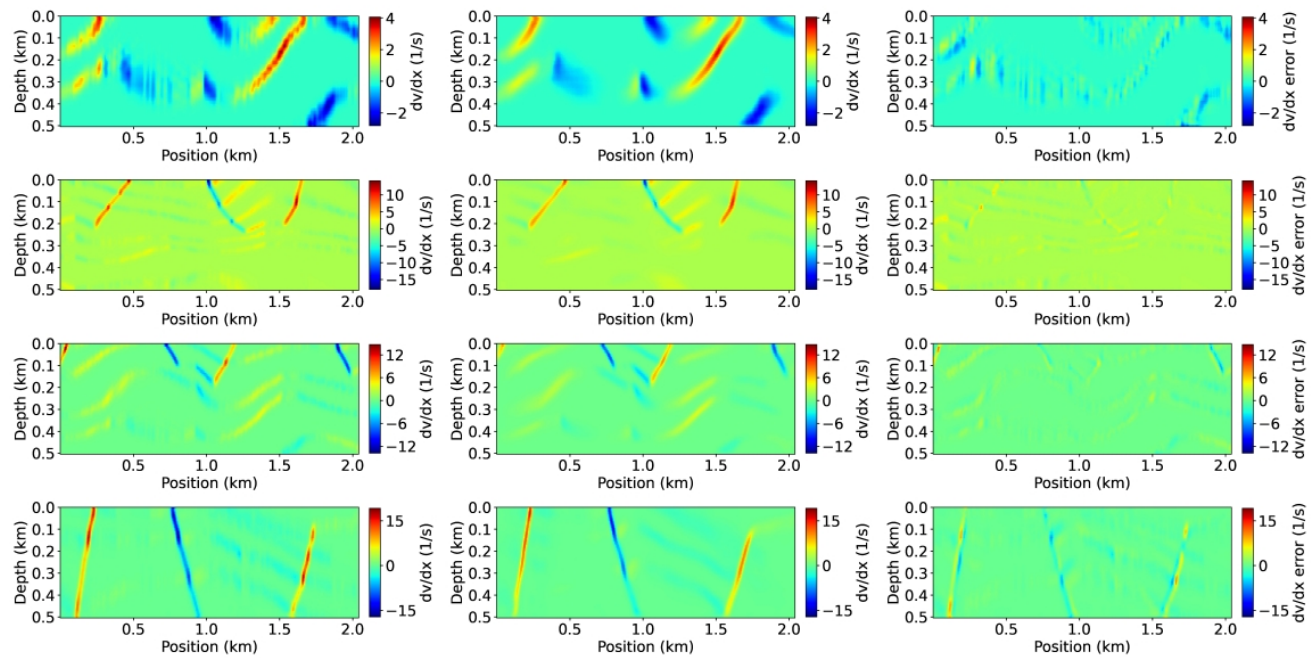

6 The first column shows four examples of true velocity derivatives (ground truth) in the horizontal direction from the test data; the second column shows the corresponding outputs of the network; the last column shows the difference between the true and the prediction velocity derivatives.

$1582 \times 794 \mathrm{~mm}(118 \times 118 \mathrm{DPI})$ 

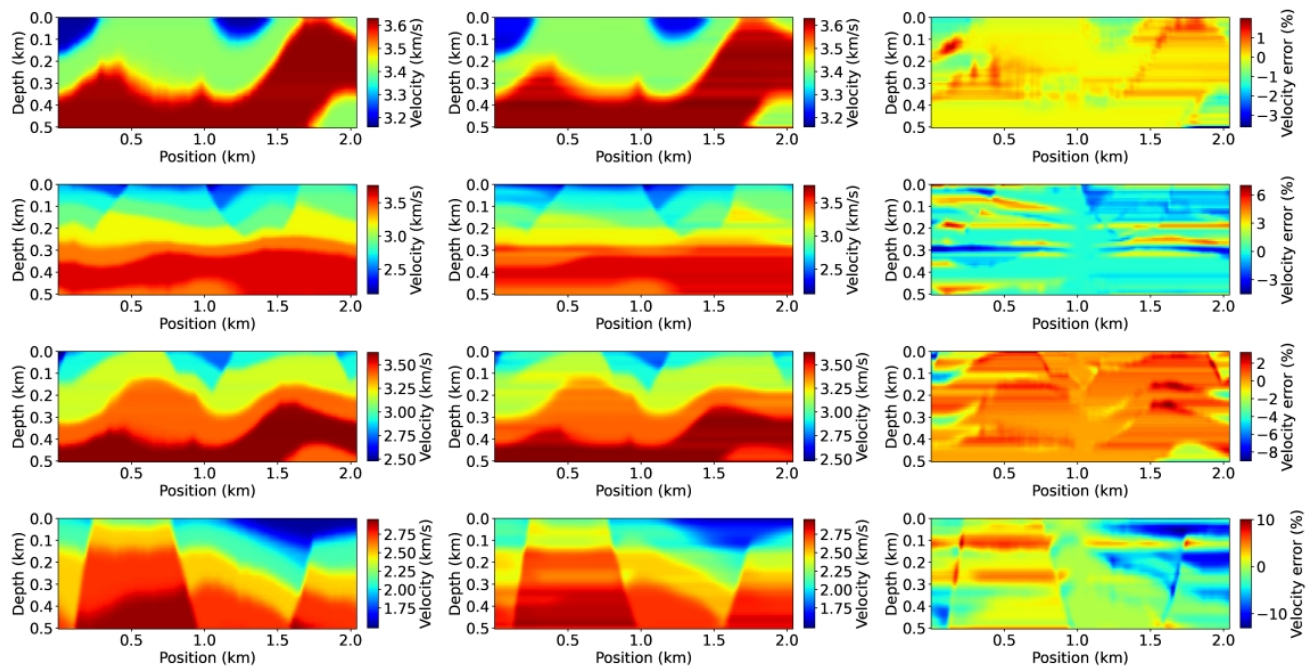

7 The first column shows true velocity models for the four selected examples in Figure 6; the second column shows the estimated velocity models built from assumed well measurements taken from the middle of the true models; the last column shows the percentage error maps between the true model and the prediction.

$1582 \times 811 \mathrm{~mm}(118 \times 118 \mathrm{DPI})$ 

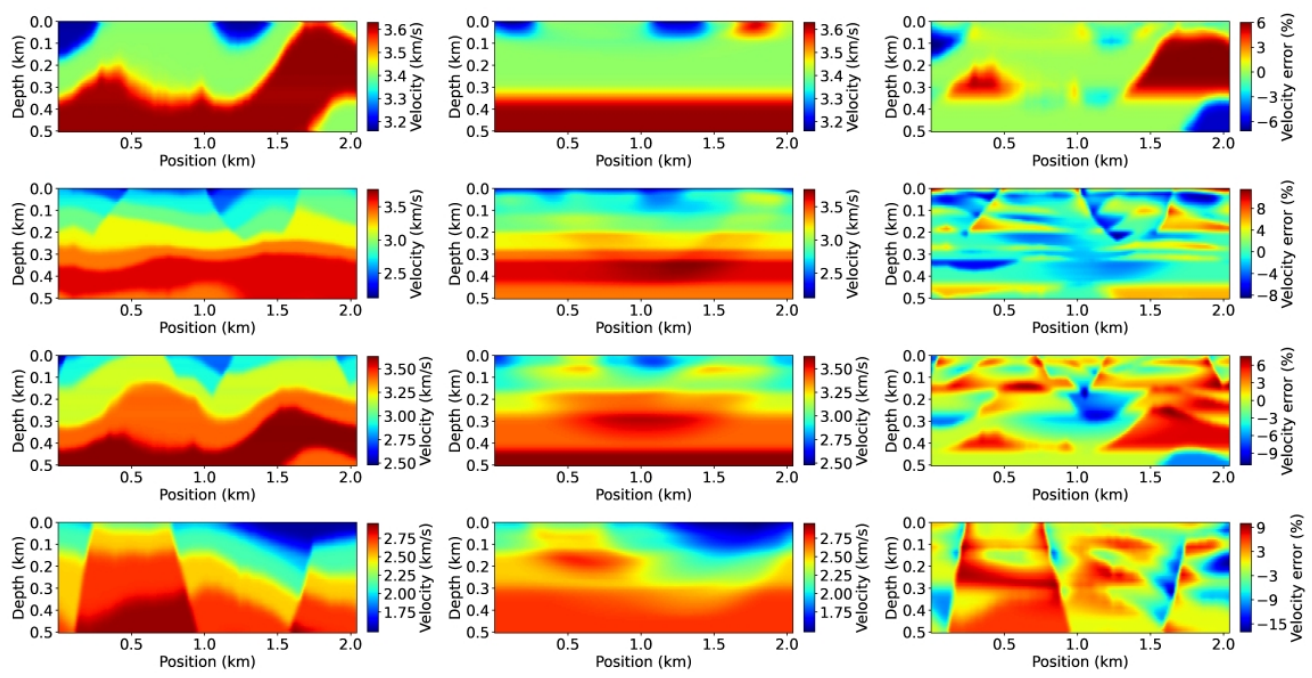

8 The first column shows true velocity models for the four selected examples in Figure 7; the second column shows estimated velocity models from conventional tomography; the last column shows the percentage error maps between the true model and the tomography result.

$1582 \times 811 \mathrm{~mm}(118 \times 118 \mathrm{DPI})$ 

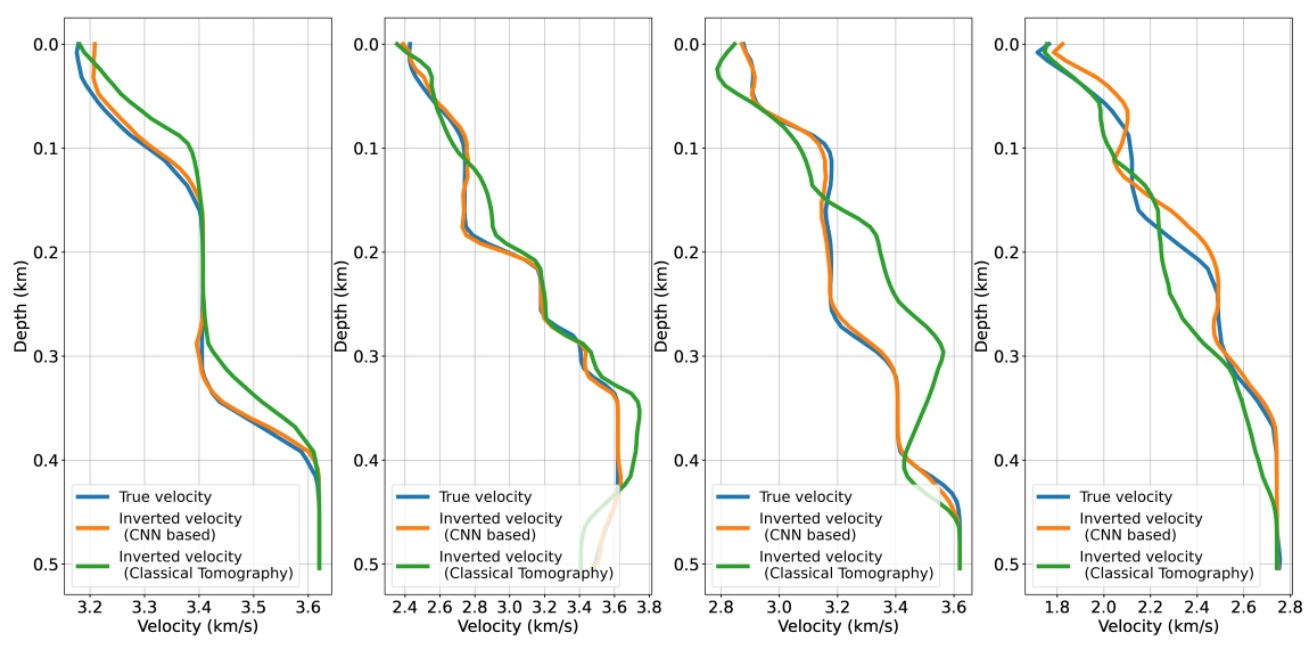

9 Vertical velocity profiles of the selected models in Figures 7 and 8 at $1.2 \mathrm{~km}$.

$1432 \times 691 \mathrm{~mm}(118 \times 118 \mathrm{DPI})$

This paper presented here as accepted for publication in Geophysics prior to copyediting and composition.

(C) 2022 Society of Exploration Geophysicists 


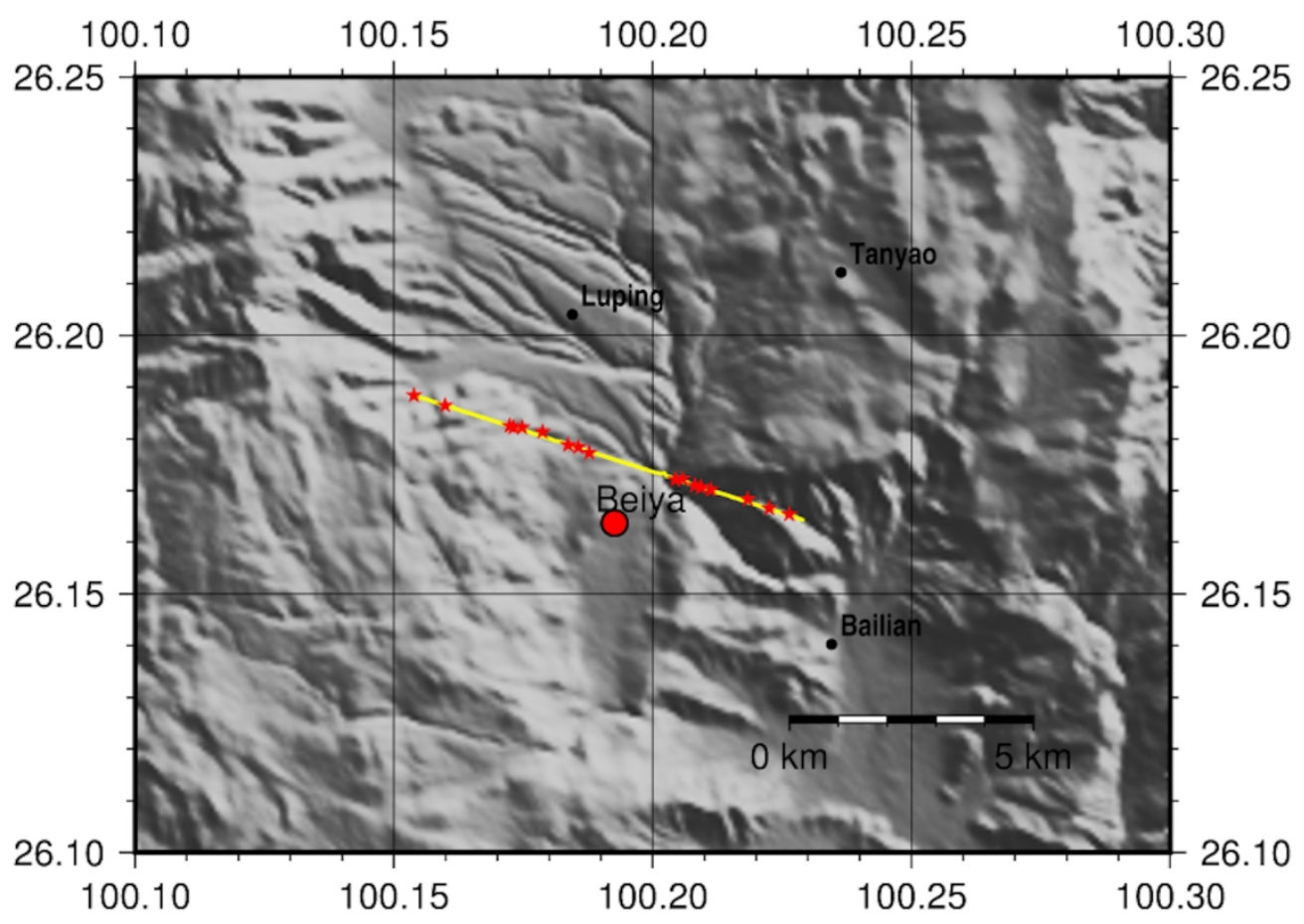

10 Location map of the study area. The yellow line is where the acquisition was conducted, and the red stars denote the shot positions (Courtesy of Kai Chang) $149 \times 105 \mathrm{~mm}(300 \times 300 \mathrm{DPI})$ 


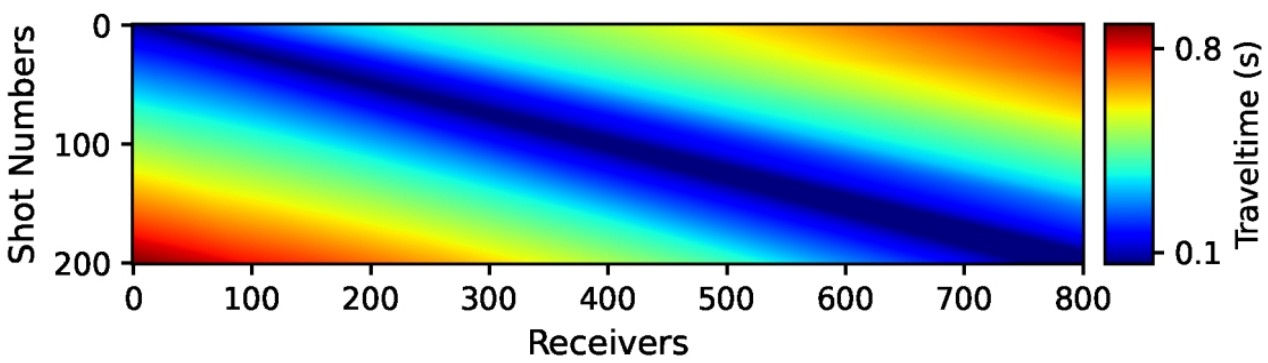

11 The interpolated traveltimes for the field data. The traveltimes were interpolated into 201 shots and 801 receivers for each shot.

$382 \times 114 \mathrm{~mm}(118 \times 118 \mathrm{DPI})$ 


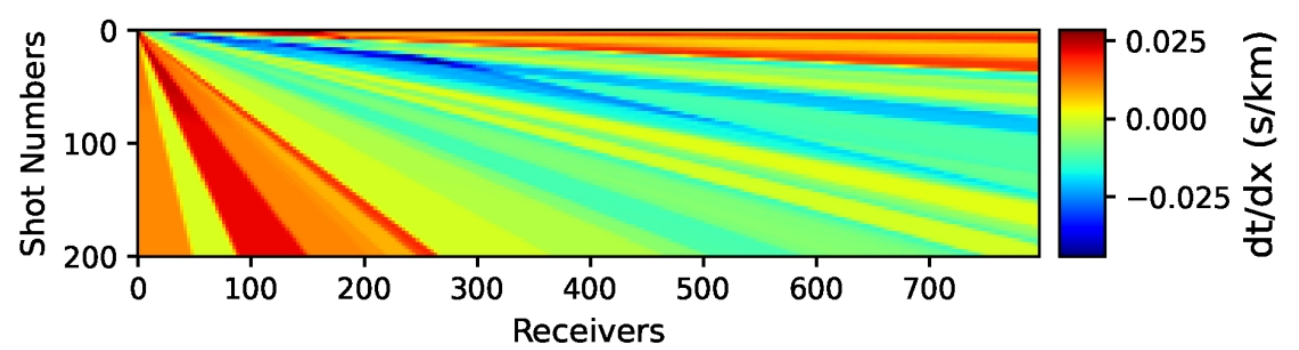

12a. The field data derivative image (a). The first arrival time derivatives computed using two successive shots form the rows of the image. CNN based predicted lateral velocity variations(b).

$404 \times 115 \mathrm{~mm}(118 \times 118 \mathrm{DPI})$ 


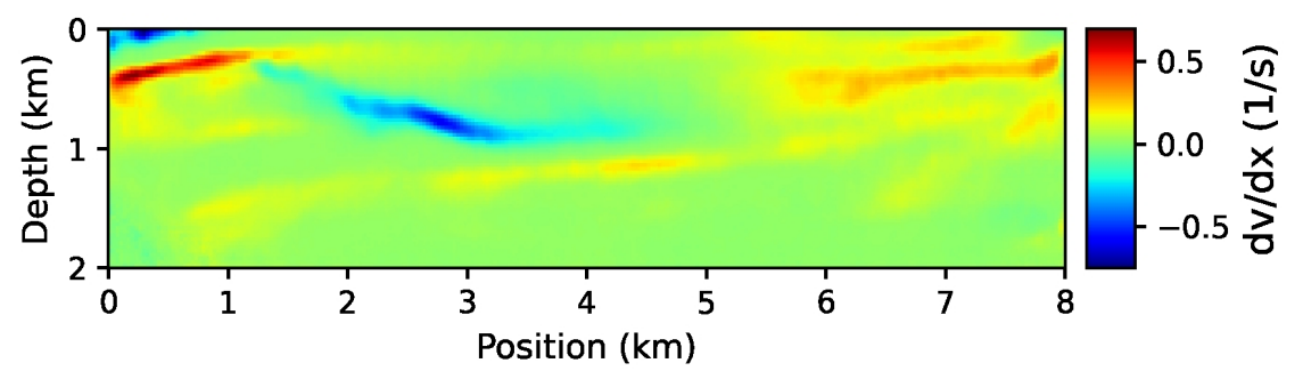

12b. The field data derivative image (a). The first arrival time derivatives computed using two successive shots form the rows of the image. CNN based predicted lateral velocity variations(b).

$381 \times 114 \mathrm{~mm}(118 \times 118 \mathrm{DPI})$ 


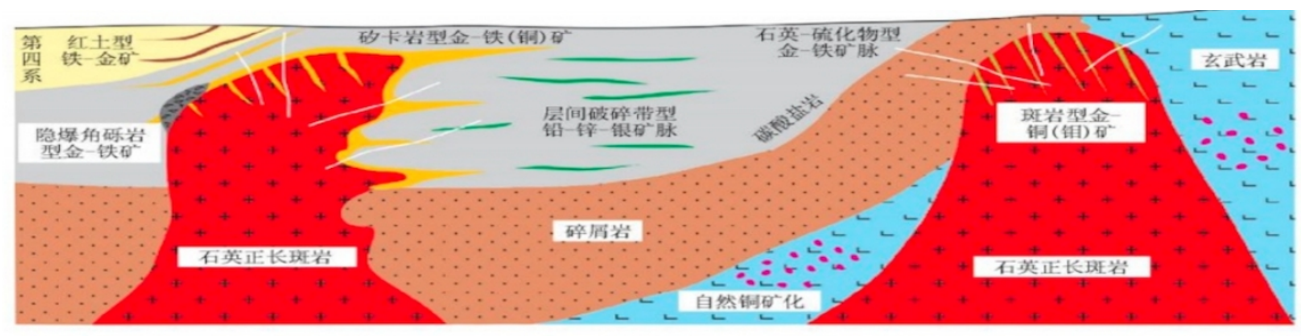

13a. A geological section representing the region of interest (Courtesy of Kai Chang, University of Science and Technology of China) (a). The inverted model from conventional tomography (b). A CNN predicted model (c).

\author{
$106 \times 26 \mathrm{~mm}(300 \times 300$ DPI)
}




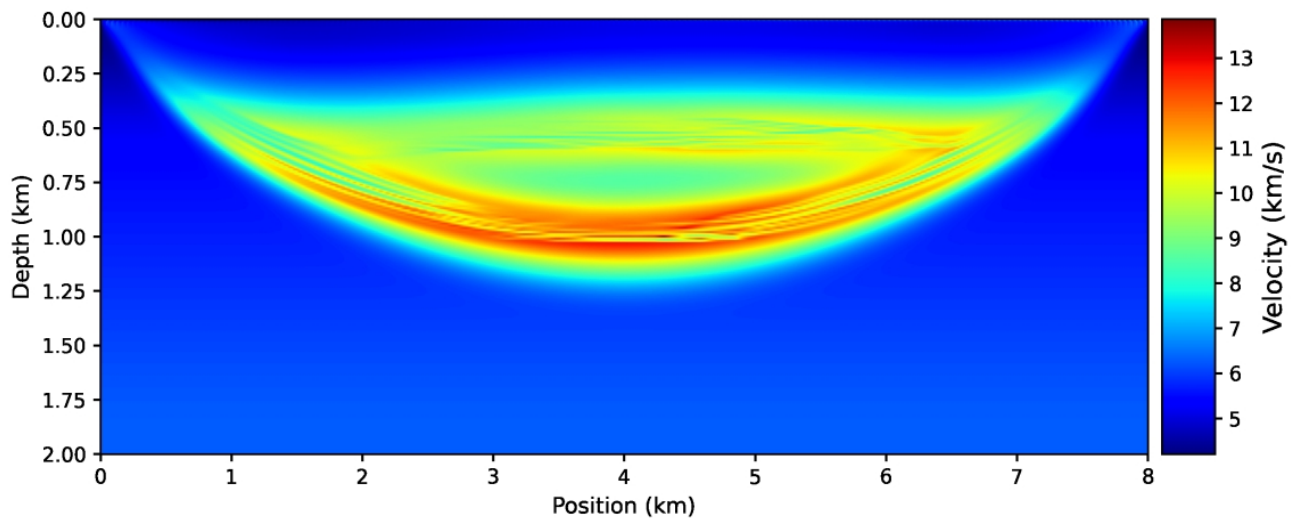

13b. A geological section representing the region of interest (Courtesy of Kai Chang, University of Science and Technology of China) (a). The inverted model from conventional tomography (b). A CNN predicted model (c).

$585 \times 240 \mathrm{~mm}(118 \times 118 \mathrm{DPI})$ 


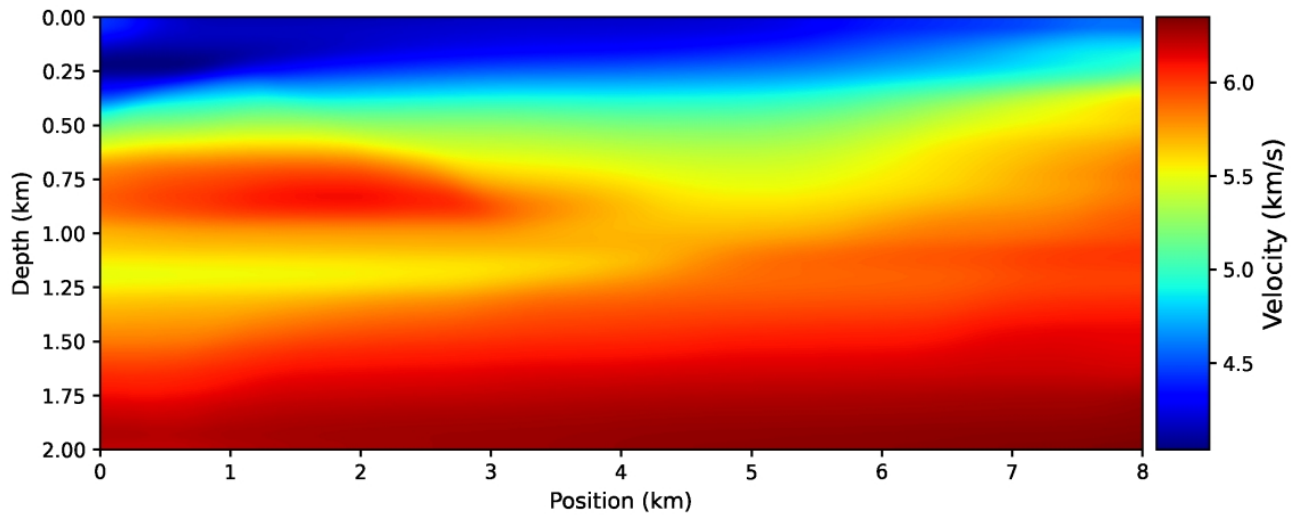

13c. A geological section representing the region of interest (Courtesy of Kai Chang, University of Science and Technology of China) (a). The inverted model from conventional tomography (b). A CNN predicted model (c).

$588 \times 240 \mathrm{~mm}(118 \times 118 \mathrm{DPI})$ 
DATA AND MATERIALS AVAILABILITY

Data associated with this research are available and can be obtained by contacting the corresponding author. 